Supporting Information for

\title{
Layer-by-Layer Self-Assembly of Polymers with Pairing Interactions
}

\author{
Gervasio Zaldivar and Mario Tagliazucchi*
}

INQUIMAE-CONICET and DQIAQF, University of Buenos Aires, School of Sciences, Ciudad

Universitaria, Pabellón 2, Ciudad Autónoma de Buenos Aires C1428EHA (Argentina).

*Email: mario@qi.fcen.uba.ar 


\section{Derivation of the Pairing Contribution to the Free Energy}

We derive here the pairing contribution to the free energy, which is based on the theory of Semenov and Rubinstein for polymer association. We modified this theory in two important aspects: i) we model the association between two different polymers, rather than the selfassociation of a single type of polymer and ii) we apply the modified Semenov-Rubinstein theory locally (i.e. we apply it at each distance from the substrate), which is justified by the fact that length scale of the features observed in the polymer concentration profiles calculated by the theory (see below) and the discretization length scale used to numerically solve the theory $(\delta=0.5 \mathrm{~nm})$ are both larger than the typical length of an $\mathrm{AB}$ bond $(\sim 0.2 \mathrm{~nm})$.

The term $\beta F_{\text {pair }}$ in the free-energy functional, eq. (2) in the main text, accounts for the free energy of pairing between poly-A and poly-B. The present work includes a polymer-polymer

pairing contribution into a molecular theory for the first time, so we will describe the derivation of this contribution in detail. Let us first write the total pairing free energy as:

$$
\frac{\beta F_{\text {pair }}}{A}=\int \beta f_{\text {pair }}(z) d z
$$

where $f_{\text {pair }}(z)$ is the paring free energy density (free energy per unit volume) for the monomers at $z$. Following Semenov and Rubinstein, ${ }^{1}$ we write the pairing free energy density as:

$$
\beta f_{\text {pair }}(z)=-\frac{1}{V} \ln \left(P_{\text {comb }}(z) \omega(z)\right)+\frac{1}{V} \beta \Delta E_{\text {pair }}^{0} N_{p}(z)
$$

where $V$ is the volume, $P_{\text {comb }}$ the number of different ways of forming $N_{\mathrm{p}}(z)$ AB pairs located at $z$ from $N_{\mathrm{A}}(z)$ monomers of type $\mathrm{A}$ and $N_{\mathrm{B}}(z)$ monomers of type $\mathrm{B}, \omega(\mathrm{z})$ is the probability that these monomers are close enough to form bonds and $\Delta E_{\text {pair }}^{0}$ is the energy of formation of $\mathrm{AB}$ pairs $\left(\Delta E_{\text {pair }}^{0}<0\right)$.

The probability $P_{\operatorname{comb}}(z)$ is:

$$
P_{\text {comb }}(z)=P_{\text {select }, A}(z) P_{\text {select }, B}(z) P_{\text {pair }}(z)
$$

where $P_{\text {select,i }}(\mathrm{i}=\mathrm{A}, \mathrm{B})$ are the different ways of selecting the $N_{\mathrm{P}}(z)$ monomers of type $\mathrm{i}$ that form $\mathrm{AB}$ pairs from the total number of monomers of type i at z, $N_{\mathrm{i}}(z)$. Thus

$$
P_{\text {select }, i}(z)=\left(\begin{array}{c}
N_{i}(z) \\
N_{p}(z)
\end{array}\right)
$$

for i $=\mathrm{A}, \mathrm{B}$. The probability $P_{\text {pair }}$ is the number of ways that the selected $N_{\mathrm{P}}(z)$ monomers of type A and $N_{\mathrm{P}}(z)$ of type $\mathrm{B}$ that can be combined: 


$$
P_{p a i r}(z)=N_{p}(z) !
$$

Combining eqs. (S3)-(S5) and expanding the combinatorial numbers yields:

$$
P_{\text {comb }}(z)=\frac{N_{A}(z) ! N_{B}(z) !}{N_{p}(z) !\left(N_{A}(z)-N_{p}(z)\right) !\left(N_{B}(z)-N_{p}(z)\right) !}
$$

The factor $\omega(\mathrm{z})$ is the probability of finding the monomers close enough to form the $\mathrm{AB}$ bonds. For given A-type monomer, the probability of finding a B-type monomer close enough to form a bond is $v_{\mathrm{AB}} / V$, where $v_{\mathrm{AB}}$ is the maximum volume that should contain both $\mathrm{A}$ and $\mathrm{B}$ in order for them to be able to pair (i.e. $v_{\mathrm{AB}}$ is approximately the volume of the $\mathrm{AB}$ pair). Thus, in order to form $N_{\mathrm{P}}(z)$ pairs:

$$
\omega(z)=\left(\frac{v_{A B}}{V}\right)^{N_{p}(z)}
$$

We can now insert eqs. (S6) and (S7) into equation (S2) to get:

$$
\begin{aligned}
& \beta f_{\text {pair }}(z)=-\left\langle n_{A}(z)\right\rangle\left(\ln \left(N_{A}(z)\right)-1\right)-\left\langle n_{B}(z)\right\rangle\left(\ln \left(N_{B}(z)\right)-1\right)+\left\langle n_{P}(z)\right\rangle\left(\ln \left(N_{p}(z)\right)-1\right) \\
& +\left(\left\langle n_{A}(z)\right\rangle-\left\langle n_{p}(z)\right\rangle\right)\left(\ln \left(N_{A}(z)-N_{p}(z)\right)-1\right)+\left(\left\langle n_{B}(z)\right\rangle-\left\langle n_{p}(z)\right\rangle\right)\left(\ln \left(N_{B}(z)-N_{p}(z)\right)-1\right) \\
& -\left\langle n_{p}(z)\right\rangle \ln \left(\frac{v_{\text {bond }}}{V}\right)+\beta \Delta E_{\text {pair }}\left\langle n_{p}(z)\right\rangle
\end{aligned}
$$

where we used Stirling's approximation for the logarithms of factorials and $\left\langle n_{i}(z)\right\rangle=N_{i}(z) / V$ for $\mathrm{i}=\mathrm{p}, \mathrm{A}$ and $\mathrm{B}$. We will define $f_{\mathrm{A}}(z)$ and $f_{\mathrm{B}}(z)$ as the fractions of monomers of type $\mathrm{A}$ and $\mathrm{B}$ that are forming pairs at $z$, namely:

$$
f_{i}(z)=\frac{N_{p}(z)}{N_{i}(z)}=\frac{\left\langle n_{p}(z)\right\rangle}{\left\langle n_{i}(z)\right\rangle}
$$

for $\mathrm{i}=\mathrm{A}, \mathrm{B}$. Replacing this definition into eq. (S8) and inserting the resulting expression for $f_{\text {pair }}(z)$ into eq. (S2) results in: 


$$
\begin{aligned}
& \frac{\beta F_{\text {pair }}}{A}=\int\left\langle n_{A}(z)\right\rangle\left(1-f_{A}(z)\right)\left(\ln \left(1-f_{A}(z)\right)\right) d z+\int\left\langle n_{A}(z)\right\rangle f_{A}\left(\ln \left(f_{A}(z)\right)\right) d z \\
& +\int\left\langle n_{B}(z)\right\rangle\left(1-f_{B}(z)\right)\left(\ln \left(1-f_{B}(z)\right)\right) d z+\int\left\langle n_{B}(z)\right\rangle f_{B}\left(\ln \left(f_{B}(z)\right)\right) d z \\
& +\int\left\langle n_{A}(z)\right\rangle f_{A}(z) \beta \Delta E_{\text {pair }}^{0} d z-\int\left\langle n_{A}(z)\right\rangle f_{A}(z)\left(\ln \left(\left\langle n_{A}(z)\right\rangle f_{A}(z) v_{A B}\right)-1\right) d z
\end{aligned}
$$

Equation (S10) is identical to eq. (3) in the main text.

\section{Minimization of the Free-Energy Functional for the Adsorption of Poly-A}

The complete free-energy functional to be minimized is:

$$
\begin{aligned}
& \frac{\beta F}{A}=\int \rho_{s}(z)\left[\ln \left(\rho_{s}(z) v_{s}\right)-1\right] d z+\int \rho_{A}(z)\left[\ln \left(\rho_{A}(z) v_{s}\right)-1\right] d z \\
& +\int \rho_{A}(z)\left[\sum_{\alpha} P_{A}(\alpha, z) \ln P_{A}(\alpha, z)\right] d z \\
& +\int\left\langle n_{A}(z)\right\rangle\left(1-f_{A}(z)\right)\left(\ln \left(1-f_{A}(z)\right)\right) d z+\int\left\langle n_{A}(z)\right\rangle f_{A}\left(\ln \left(f_{A}(z)\right)\right) d z \\
& +\int\left\langle n_{B}(z)\right\rangle\left(1-f_{B}(z)\right)\left(\ln \left(1-f_{B}(z)\right)\right) d z+\int\left\langle n_{B}(z)\right\rangle f_{B}\left(\ln \left(f_{B}(z)\right)\right) d z \\
& +\int\left\langle n_{A}(z)\right\rangle f_{A}(z) \beta \Delta E_{\text {pair }}^{0} d z-\int\left\langle n_{A}(z)\right\rangle f_{A}(z)\left(\ln \left(\left\langle n_{A}(z)\right\rangle f_{A}(z) v_{A B}\right)-1\right) d z
\end{aligned}
$$

The equilibrium state of the system results for the minimization of the total free energy, eq. (S11), subjected to two constraints. The first restriction is the packing constraint, which enforces that the sum of the volumes of all species at a given position is exactly equal to the available volume:

$$
\left\langle n_{A}(z)\right\rangle v_{p}+\left\langle n_{B}(z)\right\rangle v_{p}+\rho_{s}(z) v_{s}=1
$$

where $v_{\mathrm{s}}$ and $v_{\mathrm{p}}$ are the molecular volumes of the solvent molecules and the polymer segments, respectively. In eq. $(\mathrm{S} 12),\left\langle n_{i}(z)\right\rangle(\mathrm{i}=\mathrm{A}, \mathrm{B})$ is the density of monomers of type $\mathrm{i}$ at $z$, given by:

$$
\left\langle n_{A}(z)\right\rangle=\left\langle n_{A}^{0}(z)\right\rangle+\int \rho_{A}\left(z^{\prime}\right) \sum_{\alpha} P_{A}\left(\alpha, z^{\prime}\right) n_{A}\left(z, z^{\prime}, \alpha\right) d z^{\prime}
$$

for $\mathrm{i}=\mathrm{A}$ and

$$
\left\langle n_{B}(z)\right\rangle=\left\langle n_{B}^{0}(z)\right\rangle
$$

for $\mathrm{i}=\mathrm{B}$. 
In eqs. (S13) and (S14), $\left\langle n_{i}^{0}(z)\right\rangle(\mathrm{i}=\mathrm{A}, \mathrm{B})$ is the density of monomers of type $\mathrm{i}$ in the multilayer before the adsorption of the current poly-A layer (these density profiles are assumed to be fixed during the adsorption process). The second term in eq. (S13) is the contribution to $\left\langle n_{A}(z)\right\rangle$ from the monomers of poly-A chains in solution. In this term, $n_{\mathrm{A}}\left(z, z^{\prime}, \alpha\right) \mathrm{d} z$ is the number of monomers that a poly-A chain with its first segment at $z$ ' has between $z$ and $z+\mathrm{d} z$ when it is in conformation $\alpha$.

The second constraint is the stoichiometry condition, i.e. the number of bound A-type and B-type segments at $z$ is the same:

$$
\left\langle n_{A}(z)\right\rangle f_{A}(z)=\left\langle n_{B}(z)\right\rangle f_{B}(z)
$$

Constraints (S12) and (S15) are enforced using Lagrange multipliers. In addition to these two constrains, we should also consider the fact that the chemical potential of poly-A chains and solvent across the system are constant and, therefore, the potential to be minimize should be grand canonical for these species. Thus, the final potential to be minimized is:

$$
\begin{aligned}
& \frac{\beta W}{A}=\frac{\beta F}{A}+\int \beta \pi(z)\left[\left\langle n_{A}(z)\right\rangle v_{p}+\left\langle n_{B}(z)\right\rangle v_{p}+\rho_{s}(z) v_{s}-1\right] d z+ \\
& \int \eta(z)\left[\left\langle n_{A}(z)\right\rangle f_{A}(z)-\left\langle n_{B}(z)\right\rangle f_{B}(z)\right] d z-\mu_{A} \int \rho_{A}(z) d z
\end{aligned}
$$

The chemical potential of the solvent is not explicitly included in eq. (S16) because (as shown in ref. 2) the presence of the packing constraint allows us to redefine all chemical potentials as exchange chemical potentials. An exchange chemical potential is the free energy cost of inserting a molecule in the system in replacement of a number of solvent molecules that occupy the same volume. From this definition, $\mu_{\mathrm{s}} \equiv 0$.

Minimization of $W$ with respect to $\rho_{\mathrm{A}}(z), \rho_{\mathrm{s}}(z), f_{\mathrm{A}}(z), f_{\mathrm{B}}(z)$ and $P_{\mathrm{A}}(\alpha, z)$ yields expressions for these functions in equilibrium. Minimization of $W$ with respect to $\rho_{\mathrm{s}}(z)$ yields,

$\rho_{s}(z) v_{s}=\exp \left(-\beta \pi(z) v_{s}\right)$

Eq. (S17) shows that the Lagrange multiplier that enforces the packing constraint, $\pi(z)$, is a position-dependent osmotic pressure. ${ }^{2,3}$

The expressions resulting from the minimization of eq. (S16) with respect to $f_{\mathrm{i}}(z)$ are:

$$
\ln \left(f_{A}(z)\right)-\ln \left(1-f_{A}(z)\right)-\left(\ln \left(\left\langle n_{A}(z)\right\rangle f_{A}(z) v_{A B}\right)\right)+\beta \Delta E_{p a i r}^{0}+\eta(z)=0
$$

for $\mathrm{i}=\mathrm{A}$ and 
$\ln \left(f_{B}(z)\right)-\ln \left(1-f_{B}(z)\right)-\eta(z)=0$

for $\mathrm{i}=$ B. Adding eqs. (S18) and (S19) in order to cancel the Lagrange multiplier $\eta(z)$ and rearranging the resulting expression yields,

$$
\frac{f_{B}(z)}{\left(1-f_{A}(z)\right)\left(1-f_{B}(z)\right)\left\langle n_{A}(z)\right\rangle}=v_{A B} \exp \left(-\beta \Delta E_{\text {pair }}^{0}\right)
$$

Let us multiply both the numerator and the denominator by $\left\langle n_{A}(z)\right\rangle$ and define the molar concentration of free (unbound) monomers as $[A(z)]=C\left\langle n_{A}(z)\right\rangle\left(1-f_{A}(z)\right)$ and $[B(z)]=C\left\langle n_{B}(z)\right\rangle\left(1-f_{B}(z)\right) \quad$ and the concentration of $\mathrm{AB}$ pairs as $[A B(z)]=C\left\langle n_{A}(z)\right\rangle f_{A}(z)=C\left\langle n_{B}(z)\right\rangle f_{B}(z)$, where $C$ is a constant that relates number densities to molar concentrations $\left(C=10^{24} \mathrm{~nm}^{3} \cdot \mathrm{dm}^{-3} / N A\right.$, with $N A$ equal to Avogadro's number, equal to $\left.6.02 \cdot 10^{23} \mathrm{~mol}^{-1}\right)$. This leads to:

$$
C \frac{[A B(z)]}{[A(z)][B(z)]}=v_{A B} \exp \left(-\beta \Delta E_{\text {pair }}^{0}\right)=K^{0}
$$

which is the equilibrium equation for the pairing reaction, eq. (1) in the main text. The constant $K^{0}$ is the equilibrium constant for the formation of an $\mathrm{AB}$ pair in the bulk.

The extremum of $W$ with respect to $P_{\mathrm{A}}(\alpha, \mathrm{z})$ results in the expression:

$$
\begin{aligned}
& P_{A}(\alpha, z)= \\
& \frac{1}{\xi(z)} \exp \left\{-\int n_{A}\left(z^{\prime}, z, \alpha\right)\left[\left(\ln \left(1-f_{A}\left(z^{\prime}\right)\right)\right)+\beta \pi\left(z^{\prime}\right) v_{p}\right] d z^{\prime}\right\}
\end{aligned}
$$

where $\xi(z)$ is the partition function for the chains with a first segment at $z$, i.e.

$$
\xi(z)=\sum_{\alpha} \exp \left\{-\int d z^{\prime} n_{A}\left(z^{\prime}, z, \alpha\right)\left[\left(\ln \left(1-f_{A}\left(z^{\prime}\right)\right)\right)+\beta \pi\left(z^{\prime}\right) v_{p}\right]\right\}
$$

It is interesting to note that the Boltzmann factor in eq (S22) depends on the function $f_{\mathrm{A}}(z)$, i.e. conformations with a high number of $\mathrm{AB}$ bonds are more probable than those with a small number. As the same time, the fraction of bound segments depends on $P(\alpha, z)$ via eqs. (S13) and (S20). Therefore, the probability distribution function of the polymer chains and the fraction of bound segments across the system are highly coupled.

Finally, minimization of $W$ with respect to $\rho_{\mathrm{A}}(\mathrm{z})$ yields the equation:

$$
\rho_{A}(z)=\xi(z) \exp \left(\beta \mu_{A}\right)
$$


The set of coupled eqs. (S12), (S15), (S17), (S21), (S22) and (S24) is discretized into a lattice and solved using numerical methods. The inputs required for the adsorption of poly-A are: $K^{0}$ (bulk equilibrium constant for pair formation), the bulk density of the polymer chains $\rho_{A}^{b u l k}$, the surface density of $B$-type groups on the surface, $\sigma_{B}^{\text {surf }}$ (which is necessary for the adsorption of the first layer of poly-A) and the number of monomers per chain, $M$. The theory also requires as an input all possible polymer conformations (e.g. sum over $\alpha$ in eq. (S13)), which we approximate by a large set of $4.8 \cdot 10^{5}$ conformations randomly generated using the Rotational Isomeric Model (RIS) ${ }^{4}$ with a segment length of $0.35 \mathrm{~nm}$. As we shown in Section 7: Effect of the Size of the Conformation Set, this size is large enough to guarantee the convergence of the structure of the multilayer. Only conformations that are self-avoiding (i.e. the polymer segments do not overlap) and that do not collide with the substrate are included in the set. The choice of nonoverlapping conformations assures that intramolecular excluded-volume repulsions are exactly considered.

Following the adsorption step, a rinsing step is required to remove the polymer chains in the bulk as well as those that are loosely bound. In order to decide which chains should be removed, we first determine the average number of $\mathrm{AB}$ bonds for each conformation of the poly-A chains being adsorbed:

$$
n_{\text {bonds }}\left(\alpha, z^{\prime}\right)=\int n\left(z, z^{\prime}, \alpha\right) f_{A}(z) d z
$$

and we then remove from the system those chains with $n_{\text {bonds }}<n_{\min }$ (this cut-off value is also an input of our theory). After the rinsing step, we use the theory to calculate the adsorption of the other polymer (poly-B in this case), followed by a rinsing step. The alternated adsorption of poly$A$ and poly-B is then performed until the adsorption of the desired number of layers is calculated.

\section{Film Thickness}

In Figure $2 b$ in the main text, we plot the film thickness as a function of the number of adsorbed layers. For films with inhomogeneous density profiles there is not an unequivocal definition of film thickness. In order to determine the film thickness after the adsorption of layer $j$, we propose to use the first moment of total volume fraction of the adsorbed layers, namely:

$$
h(j)=2 \frac{\int z \sum_{k=1, j}\left\langle\phi_{k}(z)\right\rangle d z}{\int \sum_{k=1, j}\left\langle\phi_{k}(z)\right\rangle d z}
$$

Note that in the simplest case where the total density profile is a step function of thickness $d$, eq. (S26) results in $h(\mathrm{j})=d$. 
Other definitions of film thickness are possible, i.e. we can use the position of the density maximum of the last adsorbed layer or the inflection point of the total volume fraction profile. Figure S1 shows that all these definitions provide similar results and predict the linear increase in film thickness with the number of adsorbed layers, although the inflection-point method fails to accurately describe the thickness of the first $\sim 5$ adsorbed layers.

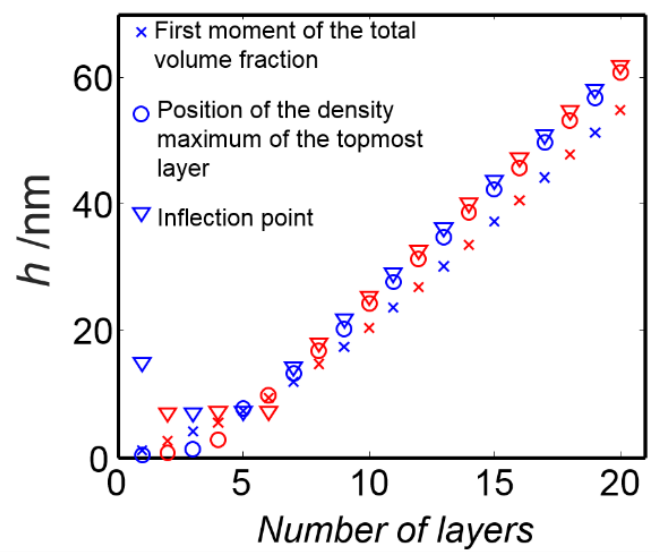

Figure S1: Film thickness as a function of the number of adsorbed layers calculated using the different methods discussed in this section. Same calculation parameters as Figure 2 in the main text.

\section{Analysis of Polymer Mixtures in Bulk Solution}

In order to explore the bulk behavior of a mixture of polymers interacting through pairing interactions, we write down the grand potential per unit of volume of a homogeneous mixture of poly-A and poly-B in solution:

$$
\begin{aligned}
& \frac{\beta W}{V}=\rho_{A}\left[\ln \left(\rho_{A} v_{s}\right)-1\right]+\rho_{B}\left[\ln \left(\rho_{B} v_{s}\right)-1\right]+\rho_{s}\left[\ln \left(\rho_{s} v_{s}\right)-1\right] \\
& +\rho_{A} M\left[\left(1-f_{A}\right)\left(\ln \left(1-f_{A}\right)\right)+f_{A}\left(\ln f_{A}\right)\right] \\
& +\rho_{B} M\left[\left(1-f_{B}\right)\left(\ln \left(1-f_{B}\right)\right)+f_{B}\left(\ln f_{B}\right)\right] \\
& -\rho_{A} M f_{A}\left(\ln \left(M \rho_{A} f_{A} v_{A B}\right)-1\right) \\
& +f_{A} \rho_{A} M \Delta E_{p a i r}^{0} \\
& +\eta\left(f_{A} \rho_{A} M-f_{B} \rho_{B} M\right) \\
& +\pi\left(\rho_{A} M v_{p}+\rho_{B} M v_{p}+\rho_{s} v_{s}-1\right) \\
& -\mu_{A} \rho_{A}-\mu_{B} \rho_{B}
\end{aligned}
$$

The potential given by eq. (S27) is analog to that used to describe polymer adsorption (eq. (S16)) with three main differences: $\mathrm{i})$ in eq. (S27), the variables $\rho_{\mathrm{i}}(\mathrm{i}=\mathrm{A}, \mathrm{B}, \mathrm{s})$ and $f_{\mathrm{i}}(\mathrm{i}=\mathrm{A}$ and B) do not depend on the spatial position within the system as we are modeling a homogeneous 
solution (while in eqs. (S11) and (S16), these functions depend on $z$ ), ii) in our mean-field approach, the polymer conformations in the bulk solution have all the same probability, thus the polymer conformational entropy becomes a constant contribution to the free energy and was not included in eq. (S27). For the same reason, in the homogeneous system the density of segments is simply $\left\langle n_{i}\right\rangle=\rho_{i} M$ (i = A, B), where $M$ is the chain length of the polymers (for simplicity, we use the same chain lengths for poly-A and poly-B), iii) while during polymer adsorption there is only one type of polymer in solution, we describe here a system where both poly-A and poly-B are present in solution and, therefore, $W$ includes the translational entropies and chemical potential contributions of both polymers. Besides these three differences, the free-energy contributions and variables in eq. (S27) are the same as those in eqs. (S11) and (S16). Note again that the chemical potential of the solvent was not included in the potential $W$ because, due to the presence of the packing constraint, the chemical potentials $\mu_{\mathrm{i}}(\mathrm{i}=\mathrm{A}, \mathrm{B})$ in the theory are exchange chemical potentials. ${ }^{2}$ An exchange chemical potential is the free energy cost of inserting a molecule of type $i$ in the system and removing an equivalent volume of water molecules. From this definition, $\mu_{\mathrm{s}} \equiv$ 0 .

The equilibrium state of the system results from the minimization of eq. (S27). Minimization with respect to $\rho_{\mathrm{s}}$ yields:

$\rho_{s} v_{s}=\exp \left(-\beta \pi v_{s}\right)$

Minimization of the potential $W$ with respect to $f_{\mathrm{i}}(\mathrm{i}=\mathrm{A}, \mathrm{B})$ yields the expressions,

$$
\ln \left(f_{A}\right)-\ln \left(1-f_{A}\right)-\left(\ln \left(\left\langle n_{A}\right\rangle f_{A} v_{A B}\right)\right)+\Delta E_{\text {pair }}^{0}+\eta=0
$$

and

$\ln \left(f_{B}\right)-\ln \left(1-f_{B}\right)-\eta=0$

which can be combined to yield,

$$
\frac{f_{B}}{\left(1-f_{A}\right)\left(1-f_{B}\right) \rho_{A} M_{A}}=K^{0}
$$

If $\rho_{\mathrm{A}}, \rho_{\mathrm{B}}$ and $K^{0}$ are known, the equilibrium eq. (S31) can be combined with the stoichiometry relation:

$$
f_{A} \rho_{A}=f_{B} \rho_{B}
$$

in order to obtain a quadratic expression that allows calculating $f_{\mathrm{A}}$ and $f_{\mathrm{B}}$.

Finally, minimization of $W$ with respect to $\rho_{i}$ yields the following expressions, 


$$
\begin{aligned}
& \mu_{A}=\ln \left(\rho_{A} v_{s}\right)+M\left[\left(1-f_{A}\right)\left(\ln \left(1-f_{A}\right)\right)+f_{A}\left(\ln f_{A}\right)\right] \\
& -M f_{A}\left(\ln \left(M \rho_{A} f_{A}\right)\right)+f_{A} M \Delta G_{\text {pair }}^{0}+\eta f_{A} M+\beta \pi M v_{p}
\end{aligned}
$$

and

$$
\begin{aligned}
& \mu_{B}=\ln \left(\rho_{B} v_{s}\right)+M\left[\left(1-f_{B}\right)\left(\ln \left(1-f_{B}\right)\right)+f_{A}\left(\ln f_{B}\right)\right] \\
& -\eta f_{B} M+\beta \pi M v_{p}
\end{aligned}
$$

These expressions can be further simplified by using eqs. (S28)-(S30) to yield:

$$
\beta \mu_{A}=\ln \left(\rho_{A} v_{s}\right)+M \ln \left(1-f_{A}\right)-\frac{M v_{p}}{v_{s}} \ln \left(\rho_{s} v_{s}\right)
$$

and

$$
\beta \mu_{B}=\ln \left(\rho_{B} v_{s}\right)+M \ln \left(1-f_{B}\right)-\frac{M v_{p}}{v_{s}} \ln \left(\rho_{s} v_{s}\right)
$$

Equations (S35) and (S36), combined with eqs. (S31) and (S32), allow to calculate the exchange chemical potentials of poly-A and poly-B as a function of the composition of the system, which is completely defined by the variables $\rho_{\mathrm{A}}, \rho_{\mathrm{B}}$ and $\rho_{\mathrm{s}}$.

We now ask the question whether the homogeneous system is thermodynamically stable or will separate into two phases of different composition. In the latter case, it should be possible to find two different compositions for the system, defined by $\rho_{A}$ ', $\rho_{B}$ ', $\rho_{s}$ ' and $\rho_{A}$ ',$\rho_{B}$ ', $\rho_{s}$ ', so that the exchange chemical potentials of $\mathrm{A}$ and $\mathrm{B}$ are equal in these phases, namely:

$$
\begin{aligned}
& \mu_{A}\left(\rho_{A}{ }^{\prime}, \rho_{B}{ }^{\prime}, \rho_{s}{ }^{\prime}\right)=\mu_{A}\left(\rho_{A}{ }^{\prime}, \rho_{B}{ }^{\prime \prime}, \rho_{s}{ }^{\prime \prime}\right) \\
& \mu_{B}\left(\rho_{A}{ }^{\prime}, \rho_{B}{ }^{\prime}, \rho_{s}^{\prime}\right)=\mu_{B}\left(\rho_{A}{ }^{\prime}, \rho_{B}{ }^{\prime \prime}, \rho_{s}{ }^{\prime \prime}\right)
\end{aligned}
$$

Note that we do not explicitly impose a similar condition on the solvent exchange chemical potential because it is always zero by definition.

We will not attempt here to explore the complete composition space for the poly-A/poly$\mathrm{B} /$ solvent ternary mixture through eqs. (S37) and (S38), but rather limit to the subset where polyA and poly-B concentrations are equal $\left(\rho_{A}=\rho_{B}\right)$. We will also assume that both polymers have the same chain length, $M$, and the same segment volume, $v_{\mathrm{p}}$. Under these highly symmetrical conditions, $\mu_{\mathrm{A}}=\mu_{\mathrm{B}}, \rho_{\mathrm{A}}{ }^{\prime}=\rho_{\mathrm{B}}{ }^{\prime}$ and $\rho_{\mathrm{A}}{ }^{\prime}{ }^{\prime}=\rho_{\mathrm{B}}{ }^{\prime}$. Figure $\mathrm{S} 2$ shows the chemical potential of poly-A and poly-B as a function of the composition of the system (which we express in terms of the total molar concentration of monomers in solution, $c_{\mathrm{p}}$ ). We observe that there are pairs of composition of equal chemical potential that fulfill eqs. (S37) and (S38), see for example red dashed line. Our model of polymers interacting through pairing attractions predicts, therefore, the possibility of 
phase separation into a polymer-rich phase and a polymer-poor phase. The composition at the local maximum of the $\mu_{\mathrm{i}}$ curve (red dot I in Figure S2) determines the onset of phase separation (i.e. for higher polymer concentrations, the homogeneous phase becomes thermodynamically unstable and phase separation must occur). This composition is given by,

$\frac{\partial \mu_{A}}{\partial c_{p}}=\frac{\partial \mu_{B}}{\partial c_{p}}=0$

It is very important to note that for a two-component system, the onset of phase separation is always given by the composition where the first derivative of the chemical potential with respect to the density of one of the components is zero (i.e. condition given in eq. (S39)). ${ }^{5}$ However, this useful condition is not generally valid for systems with three or more components and thus phase separation in such cases should be proved by finding the compositions that fulfill equations (S37) -(S38). ${ }^{6,7}$ In the present case, however, we can use eq. (S39) for a three-component system only because we have required highly symmetrical conditions on the densities $\left(\rho_{A}=\rho_{B}\right)$, chain lengths and segment volumes.

Using eq. (S39), we have determined the limit of thermodynamic stability of the homogeneous phase as a function of the pairing constant and chain length, which are shown in Figure 1 in the main text. 


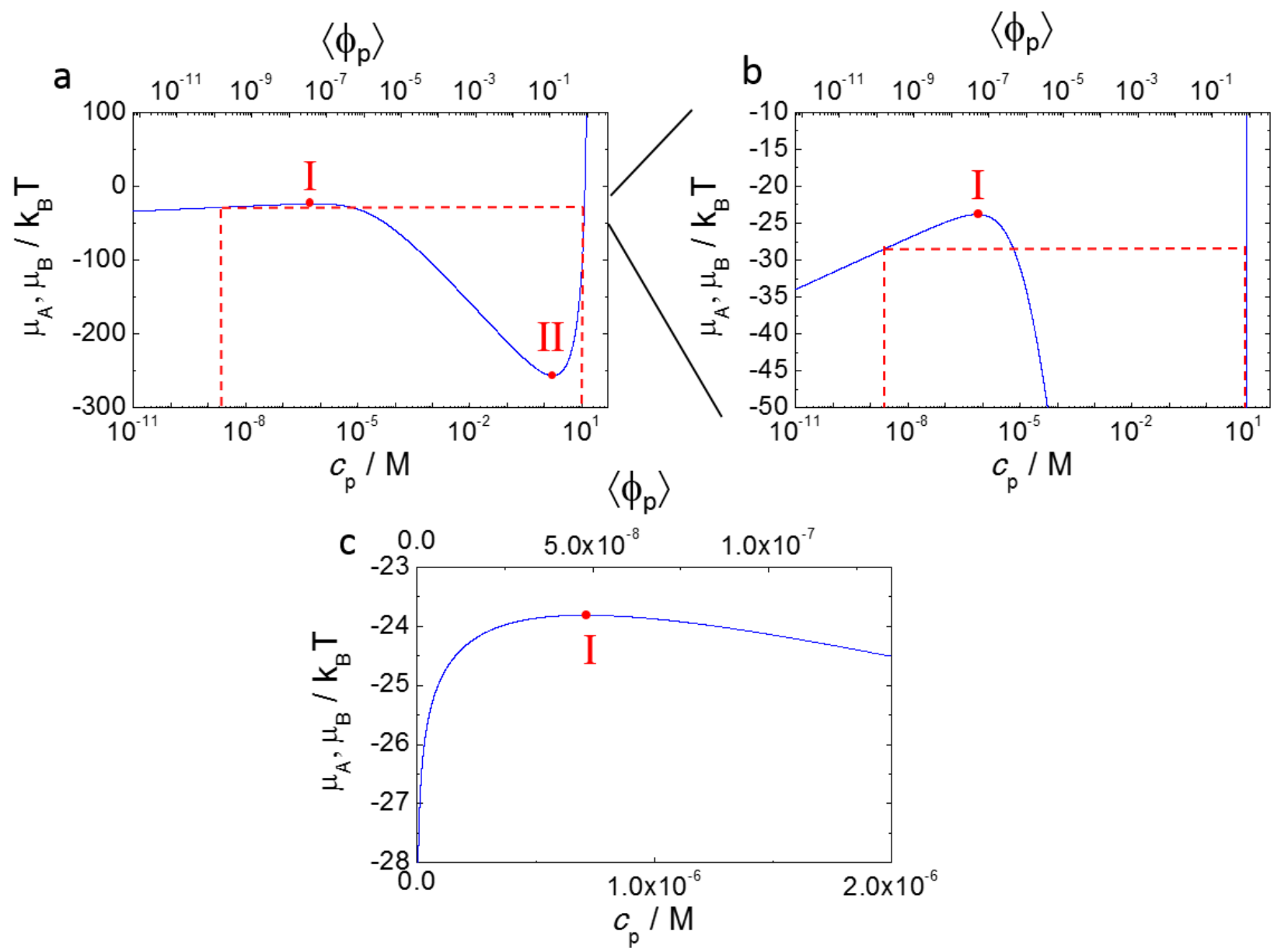

Figure S2: Chemical potential of poly-A and poly-B as a function of the total concentration of polymer in the system ( $c_{\mathrm{p}}$, expressed as molar concentration of monomers). The upper $\mathrm{x}$-axis shows the total volume fraction of the polymer in the system, $\left\langle\phi_{\mathrm{p}}\right\rangle$. Panels $\mathrm{b}$ and $\mathrm{c}$ show a zoom of the curve in panel a. The red points I and II show the compositions at the onsets of phase separation, between these two points, the homogeneous phase is thermodynamically unstable and phase separation must occur. The red dashed lines in panels a and $\mathrm{b}$ show two different compositions that have the same chemical potentials for poly-A and poly-B and that correspond to chemical compositions of coexisting phases in the phase-separated system. Calculations conditions: $K^{0} / C=$ $6.8 \cdot 10^{3} \mathrm{M}^{-1}, M=50$.

\section{Minimum Value of $M$ that Allows Phase Separation.}

A very interesting prediction of our theory is that phase separation is not observed when $M$ $\leq 2$. For example, Figure S3 shows that the chemical potentials for $M=3$ and 4 have a maximum as a function of $c_{\mathrm{p}}$, while that for $M=2$ is a monotonically increasing function of $c_{\mathrm{p}}$ (this behavior is observed in the whole range of $c_{\mathrm{p}}$ and $K^{0}$ explored). Therefore, the system for $M=2$ is always homogeneous (at least when $\rho_{\mathrm{A}}=\rho_{\mathrm{B}}$ ). In order to understand this behavior, we can analytically explore the limit $K^{0} \rightarrow \infty\left(f_{\mathrm{A}}, f_{\mathrm{B}} \rightarrow 1\right)$ since the stability of the phase-separated region in the phase 
diagram monotonically increases with increasing $K^{0}$ (see Figure 1 in the main text). Due to the highly symmetrical conditions imposed to the concentrations, we also have $f_{\mathrm{A}}=f_{\mathrm{B}}$. Using these results, we can approximate eq. (S31) to,

$$
\frac{1}{\left(1-f_{A}\right)^{2} \rho_{A} M}=K^{0} \quad \text { for } f_{\mathrm{A}}, f_{\mathrm{B}} \rightarrow 1
$$

Isolating $\left(1-f_{\mathrm{A}}\right)$ from eq. (S40) and inserting the result into eq. (S35) yields,

$$
\mu_{A}=\ln \left(\rho_{A} v_{s}\right)-\frac{M}{2}\left[\left(\ln \left(K^{0} \rho_{A} M\right)\right)\right]-\frac{M v_{p}}{v_{s}} \ln \left(1-2 \rho_{A} M v_{p}\right)
$$

where we have used the packing constraint

$$
\rho_{A} M v_{p}+\rho_{B} M v_{p}+\rho_{s} v_{s}=1
$$

and the fact that $\rho_{A}=\rho_{B}$ to replace $\rho_{\mathrm{s}} v_{\mathrm{s}}$ in the last term of eq. (S35). Differentiation of eq. (S41) with respect to the density of the polymer yields,

$$
\frac{\partial \mu_{A}}{\partial \rho_{A}}=\frac{1}{\rho_{A}}-\frac{M}{2} \frac{1}{\rho_{A}}+\frac{M v_{p}}{v_{s}} \frac{2 M v_{p}}{1-2 \rho_{A} M v_{p}}
$$

The requirement for thermodynamic instability of the homogeneous phase is $\frac{\partial \mu_{A}}{\partial \rho_{A}}<0$. The first and third terms in Eq. (S43) (arising from the translational entropy of the polymers and the osmotic pressure) are always positive, thus favor the homogeneous system. The second term (arising from pairing interactions) is always negative and favors phase separation. For typical values of $\rho_{\mathrm{A}}(<$ $10^{-3} \mathrm{~nm}^{-3}$ ), the third term is much smaller than the first one, so we can neglect it and study the competition between translational entropy of the chains and pairing interactions, which yields,

$$
\frac{1}{\rho_{A}}-\frac{M}{2 \rho_{A}}<0
$$

Therefore, the necessary condition for phase separation is $M>2$, in agreement with the numerical results in Figure 1 of the main text. If the third term of equation (S43) is included into the analysis, the minimum $M$ that allows phase separation will depend on the concentration of polymer, but it will be always larger than two. 


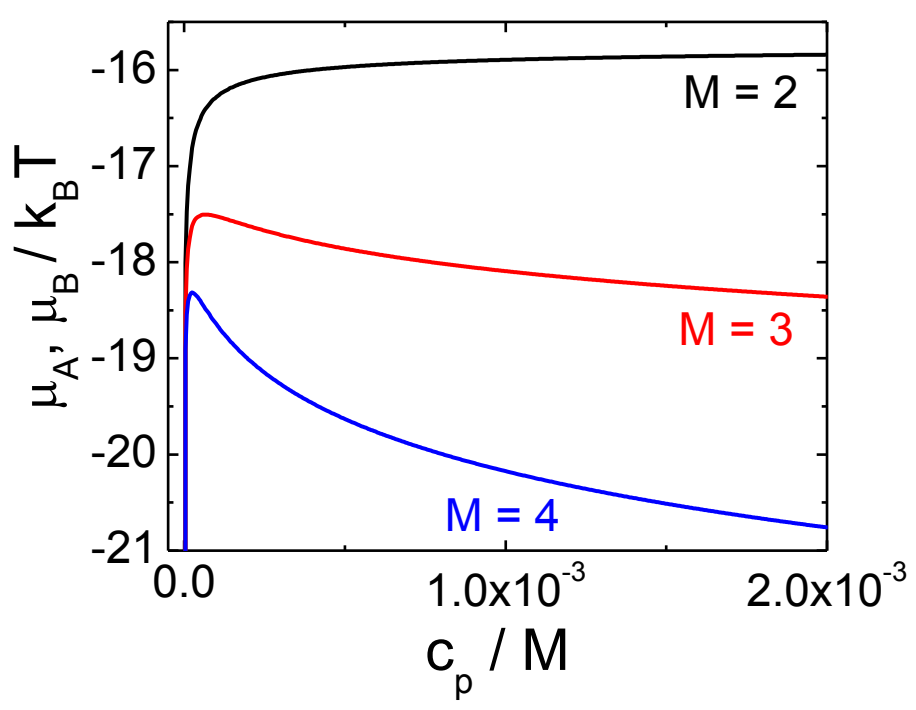

Figure S3: Same as Figure S2c for different values of $M$ and $K^{0}=6.8 \cdot 10^{3} \mathrm{M}^{-1}$.

\section{Derivation of Equation 6 in the main text}

In order to derive eq. (6) in the main text, let us consider the adsorption of a poly-A layer on top of the 20-layer poly-B-capped multilayer shown in Figure 2a. Figure S4 shows the volume fraction profile of the excess of B-type monomers, $\left\langle\phi_{\mathrm{B}}(z)\right\rangle^{\mathrm{exc}}$, which we define as the difference between the total volume fraction of B-type and A-type monomers before the adsorption of an additional layer of poly-A, i.e..

$$
\left\langle\phi_{B}(z)\right\rangle^{e x c}=\sum_{j=2,4, \ldots, 20}\left\langle\phi_{j}(z)\right\rangle-\sum_{j=1,3, . ., 19}\left\langle\phi_{j}(z)\right\rangle
$$

The Figure also shows the volume fraction profile of A-type monomers, $\left\langle\phi_{\mathrm{A}}(z)\right\rangle^{\text {ads }}$, resulting from the adsorption of the new poly-A layer (layer 21). Both profiles exhibit a peak near the film/solution interface, thus, in order to construct a simple argument, we will consider only the peak values at the interface, $\left\langle\phi_{\mathrm{B}}\right\rangle^{\mathrm{exc}}$ and $\left\langle\phi_{\mathrm{A}}\right\rangle^{\mathrm{ads}}$, rather that the complete $\mathrm{z}$-dependent volume fraction profile. We can write the chemical potential of poly-A at the interface using eq. (S35) as,

$$
\beta \mu_{A}^{i}=\ln \left(\frac{\left\langle\phi_{A}\right\rangle^{a d s} v_{s}}{v_{p} M}\right)+M\left[\left(\ln \left(1-f_{A}\right)\right)\right]-\frac{M v_{p}}{v_{s}} \ln \left(\left\langle\phi_{s}\right\rangle\right)
$$


where we used $\left\langle\phi_{s}\right\rangle=\rho_{s}^{i} v_{s}$ and $\left\langle\phi_{A}\right\rangle^{a d s}=\rho_{A}^{i} v_{p} M$ and $\rho_{\mathrm{A}}{ }^{\mathrm{i}}$ and $\rho_{\mathrm{s}}{ }^{\mathrm{i}}$ are the number density of polymer chains and solvent at the interface, respectively. We then replace $\left\langle\phi_{s}\right\rangle$ from the packing constraint (eq. (S42)) and $f_{\mathrm{A}}$ from the stoichiometry condition, eq. (S32), to get:

$\beta \mu_{A}^{i}=\ln \left(\frac{\left\langle\phi_{A}\right\rangle^{a d s} v_{s}}{M v_{p}}\right)+M \ln \left(1-\frac{\left\langle\phi_{B}\right\rangle^{e x c} f_{B}}{\left\langle\phi_{A}\right\rangle^{a d s}}\right)-\frac{M v_{p}}{v_{s}} \ln \left(1-\left\langle\phi_{A}\right\rangle^{a d s}-\left\langle\phi_{B}\right\rangle^{e x c}\right)$

In our symmetric system, the multilayer can grow linearly only if the excess of A-type segments after the adsorption of the new poly-A layer is exactly the same as the excess of B-type segments before adsorption. Therefore, the total number of A-type segments in the newly adsorbed poly-A layer have to be exactly twice the number of the excess B-type segments before the adsorption (see volume fraction profiles in Figure S4). From this argument, $\left\langle\phi_{\mathrm{B}}\right\rangle^{\text {exc }} \sim 1 / 2\left\langle\phi_{\mathrm{A}}\right\rangle^{\mathrm{ads}}$.

We will also use the fact that after the adsorption of poly-A there is an excess of A-type groups at the interface, and, therefore, $f_{\mathrm{B}} \sim 1$. Applying these arguments to eq. (S47) results in:

$\beta \mu_{A}^{i}=\ln \left(\frac{\left\langle\phi_{A}\right\rangle^{a d s} v_{s}}{M v_{p}}\right)+M \ln \left(\frac{1}{2}\right)-\frac{M v_{p}}{v_{s}} \ln \left(1-\frac{3}{2}\left\langle\phi_{A}\right\rangle^{a d s}\right)$

On the other hand, in the bulk solution, $\left\langle\phi_{\mathrm{s}}\right\rangle \sim 1$ and $f_{\mathrm{A}}=0$, so evaluating eq. (S35) in the bulk solution yields,

$\beta \mu_{A}^{b u l k}=\ln \left(\frac{\left\langle\phi_{A}\right\rangle^{b u l k} v_{s}}{M v_{p}}\right)$

where $\left\langle\phi_{\mathrm{A}}\right\rangle^{\text {bulk }}$ is the volume fraction of poly-A in the bulk solution. Equating the chemical potentials of eqs. (S48) and (S49), finally leads to,

$\ln \left(\frac{\left\langle\phi_{A}\right\rangle^{a d s}}{\left\langle\phi_{A}\right\rangle^{b u l k}}\right)+M\left[\left(\ln \left(\frac{1}{2}\right)\right)\right]-\frac{M v_{p}}{v_{s}} \ln \left(1-\frac{3}{2}\left\langle\phi_{A}\right\rangle^{a d s}\right)=0$

which is equation 6 in the main text. 


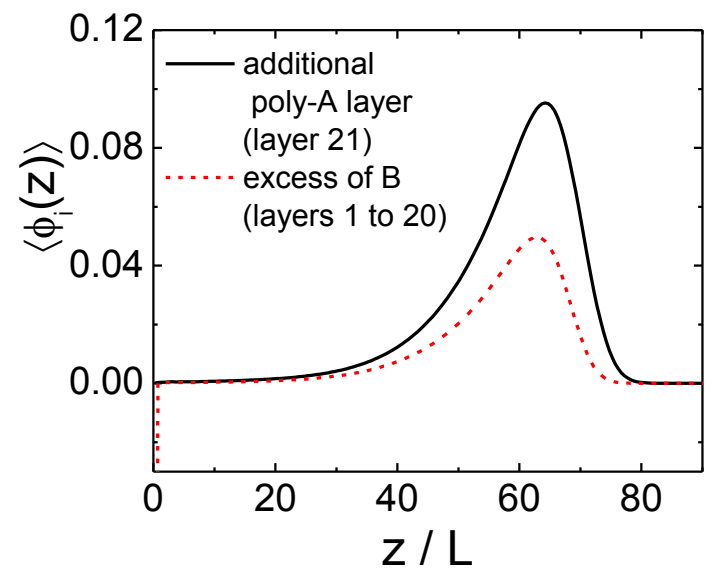

Figure S4: Volume fraction profile for the excess of $\mathrm{B},\left\langle\phi_{\mathrm{B}}(z)\right\rangle^{\text {exc }}$ (see eq. (S45)) for a 20-layer film capped with poly-B (same system as in Figure 2a in the main text) and for an additional poly-A layer deposited on top of this multilayer. Same calculation parameters as in Figure 2a in the main text.

\section{Effect of the Size of the Conformation Set}

Figure S5 shows the volume fraction profiles for all layers in the film for two different sizes of the set of polymer conformations that is required as an input for the theory (see Section 2). The profiles for the set of $4.8 \cdot 10^{5}$ conformations (red lines, set used for the calculations shown in the main text) and $9.6 \cdot 10^{5}$ conformations (blue lines) are very similar, thus confirming that our results are converged with respect to the size of the set of conformations.

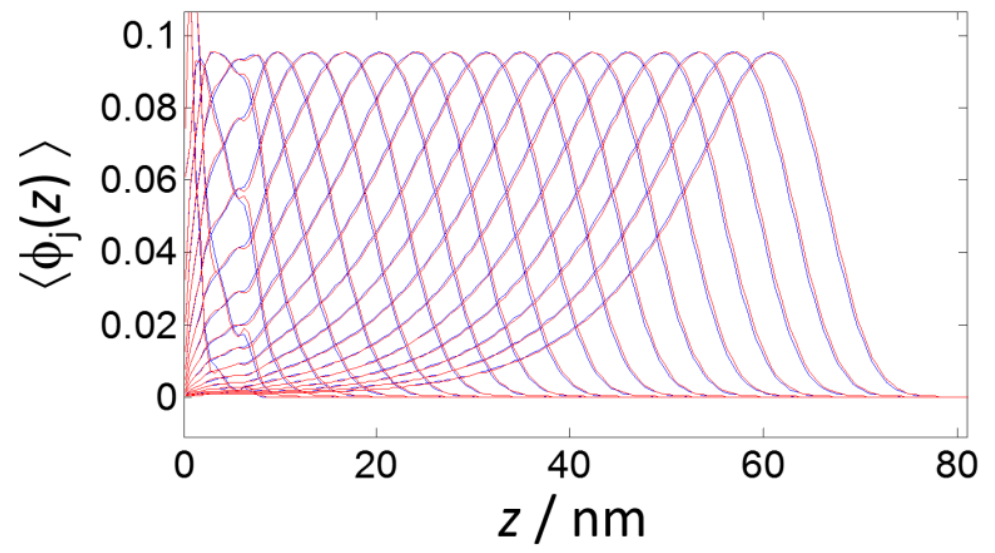

Figure S5: Predicted volume fraction of layer $\mathrm{j},\left\langle\phi_{\mathrm{j}}\right\rangle$, as a function of the distance from the substrate for the LbL adsorption of 20 layers (terminated in poly-B) for two different sizes of the set of chain conformations: $4.8 \cdot 10^{5}$ (red lines) and $9.6 \cdot 10^{5}$ (blue lines) conformations. Same calculation conditions as in Figure 2 in the main text. 


\section{References}

1. Semenov, A. N.; Rubinstein, M. Macromolecules 1998, 31, 1373-1385.

2. Nap, R.; Gong, P.; Szleifer, I. J. Polym. Sci., Part B: Polym. Phys. 2006, 44, 2638-2662.

3. Szleifer, I.; Carignano, M. A. Adv. Chem. Phys. 1996, 96, 165-260.

4. $\quad$ Flory, P. J., Statistical mechanics of chain molecules. Wiley: New York, 1969.

5. Flory, P. J. J. Chem. Phys. 1942, 10, 51-61.

6. Scott, R. L. J. Chem. Phys. 1949, 17, 279-284.

7. Hsu, C. C.; Prausnitz, J. M. Macromolecules 1974, 7, 320-324. 